\title{
Salvianolic Acid B-Loaded Chitosan/hydroxyapatite Scaffolds Promotes The Repair Of Segmental Bone Defect By Angiogenesis And Osteogenesis
}

This article was published in the following Dove Press journal: International Journal of Nanomedicine

\section{Chuanlei Ji* \\ Long $\mathrm{Bi}^{*}$ \\ Jing $\mathrm{Li}$ \\ Junjun Fan}

Department of Orthopedics Surgery,

Xijing Hospital, Air Force Medical

University, Xi'an 710032, People's

Republic of China

*These authors contributed equally to this work
Correspondence: Jing Li; Junjun Fan Department of Orthopedics Surgery,

Xijing Hospital, Air Force Medical

University, Xi'an 710032, People's

Republic of China

Email zyfmmu@fmmu.edu.cn;

fanjunjunys@I63.com
Background: Salvianolic acid B has been proven as an effective drug to promote osteogenesis and angiogenesis which could be beneficial for bone repair.

Purpose: The objective of this study was to construct a salvianolic acid B-loaded chitosan/ hydroxyapatite (Sal B-CS/HA) bone scaffold with controlled release and effective bioactivity.

Methods: The characteristics, controlled release behavior and bioactivity of Sal B-CS/HA scaffold were evaluated in vitro. The bone repair effect was evaluated in the rabbit radius defect model.

Results: The results showed that chemical and physical characteristics of salvianolic acid $\mathrm{B}$ and chitosan/hydroxyapatite (CS/HA) material did not obviously change after the drug loading procedure; the drug release of salvianolic acid B was stable and continuous from the Sal BCS/HA scaffold for 8 weeks in vitro; the biocompatibility of the Sal B-CS/HA was favorable by evaluation of cell morphology and proliferation; the osteogenic and angiogenic bioactivities of the Sal B-CS/HA scaffold were proved to be effective by in vivo and in vitro tests.

Conclusion: Our results suggest that this salvianolic acid B-loaded bone scaffold has potential to be used for bone defect repair with both osteogenic and angiogenic bioactivities. Keywords: angiogenesis, bone tissue engineering, controlled release, drug-loaded bone scaffold, osteogenesis, salvianolic acid B

\section{Introduction}

The repair of large bone defect remains a great challenge and the bone tissue engineering represents one of the most promising methods for solving this problem. ${ }^{1}$ The implantation of biomaterials which can load bioactive agents and release the agents locally in the defect area is proven as an effective approach., ${ }^{2,3}$ However, the cells seeded on the scaffold can only get the oxygen and nutrients from the scaffold surface with a distance of $200 \mu \mathrm{m} .{ }^{4}$ So, the interior of the scaffold lacks blood vessel network and the bone formation is limited by the vascularization of the scaffold after grafted into the body. ${ }^{5}$ Therefore, to get large size of tissueengineered bone grafts (TEBG), the drug-loaded scaffold should load the agents which have the ability of promoting osteogenesis and vascularization.

Salvianolic acid B (molecular formula: $\mathrm{C}_{36} \mathrm{H}_{30} \mathrm{O}_{16}$, molecular weight: 718.62) is one of the most effective organic acids extracted from salvia miltiorrhiza. And, salvianolic acid B has the angiogenic bioactivity of protecting the vascular endothelium cells and promoting the proliferation of vascular endothelium cells. ${ }^{6-8}$ Recent $^{-}$ studies suggest that salvianolic acid B can also promote the osteoblasts proliferation and osteogenesis. ${ }^{9-11}$ But there was little report about the salvianolic acid B-loaded 
scaffold with satisfying release behavior and good bioactivity. So, we tried to construct a drug-loaded scaffold with the controlled release of salvianolic acid B to utilize its osteogenic and angiogenic bioactivities.

The ideal drug-loaded bone scaffold should have good biocompatibility and good release behavior. ${ }^{12,13}$ Hydroxyapatite (HA) is a natural component of bone and has been used widely for bone scaffold. ${ }^{14,15}$ To overcome the brittleness of HA, chitosan (CS) has been used to combine with HA for its excellent biocompatibility and biodegradability. ${ }^{16,17} \mathrm{CS}$ is also considered as an ideal drug vehicle to be widely used in drug delivery system. ${ }^{18,19}$ In our previous study, the chitosan/hydroxyapatite (CS/HA) scaffold combined the advantages of both $\mathrm{CS}$ and $\mathrm{HA}$ is proved to be a promising bone scaffold and drug release system. ${ }^{20}$

In this study, we developed a salvianolic acid B-loaded chitosan/hydroxyapatite (Sal B-CS/HA) scaffold which could release the salvianolic acid B steadily and durably. And, the scaffold characteristics, controlled release behavior and bioactivity were evaluated in vitro. The bone defect repair effect was evaluated in vivo. We hypothesized the incorporation of salvianolic acid B could promote the effect of chitosan/hydroxyapatite scaffold on the repair of segmental bone defect through the osteogenesis and angiogenesis effect of salvianolic acid B.

\section{Materials And Methods Preparation Of Scaffolds}

Salvianolic acid B (molecular formula: $\mathrm{C}_{36} \mathrm{H}_{30} \mathrm{O}_{16}$, molecular weight: 718.62 , standard quality) was purchased from the Sima-Lab Company (Tianjin, China); CS (molecular weight $=2.5 \times 10^{5}$, degree of deacetylation $\geq 90.0 \%$, viscosity $>100 \mathrm{cps}$, biomedical grade) was purchased from the Pioneer Biotech Company (Xi'an, China). At first, $2 \mathrm{~g}$ chitosan was weighed and added into $100 \mathrm{~mL} 2 \%(\mathrm{v} / \mathrm{v})$ acetic acid, and the CS solution was stirred for $20 \mathrm{mins}$. Then, 36mg salvianolic acid B was dissolved in $1 \mathrm{~mL}$ ethanol and added to chitosan solution and stirred for 2hrs. Then, $\mathrm{Ca}\left(\mathrm{NO}_{3}\right)_{2}$ and $\mathrm{KH}_{2} \mathrm{PO}_{4}(\mathrm{Ca} / \mathrm{P}=1.67)$ were added slowly to prepare the Sal B-CS/HA precursor solution (final mass ratio of CS/HA was $1 / 2$ ). Then, the precursor solution was homogenized for $4 \mathrm{hrs}$ by vigorous stirring and centrifuged for $10 \mathrm{mins}$ to remove the air bubbles. The solution was poured into a plastic mold $\left(4.0 \mathrm{~mm}\right.$ in diameter, $20 \mathrm{~mm}$ in length) and placed at $4^{\circ} \mathrm{C}$ for $2 \mathrm{hrs}$ and $-10^{\circ} \mathrm{C}$ for $3 \mathrm{hrs}$, then the solution was freeze-dried for 24hrs. After they were fully dried, Sal B-CS/HA scaffolds were soaked in $2 \%(\mathrm{wt} / \mathrm{v}) \mathrm{NaOH}$ for $2 \mathrm{hrs}$ and rinsed with distilled water for several times. After that, scaffolds were freeze-dried again, then both ends of the scaffold were truncated for $2.5 \mathrm{~mm}$. Finally, each Sal B-CS/HA scaffold contained $10^{-7} \mathrm{~mol}$ salvianolic acid B. We also prepared the CS/HA scaffolds in the same method without salvianolic acid B as the control group. All scaffolds were sterilized with $20 \mathrm{kGy}{ }^{60} \mathrm{Co}$ and stored in vacuum packages at room temperature before subsequent use.

\section{Characterization Of Scaffolds} The Microstructure And Phase Of The Scaffolds

The microstructure of Sal B-CS/HA scaffolds was examined by scanning electron microscopy and HE staining. Infrared absorption spectra of salvianolic acid B, CS/HA and salvianolic acid B-CS/HA were recorded by Fourier transform infrared spectroscopy in the $4000-400 \mathrm{~cm}^{-1}$ range. One scaffold from each group was grinded into powder and dried at $50^{\circ} \mathrm{C}$ over $24 \mathrm{hrs}$. Potassium bromide was baked in agate mortar under the infrared light for $3 \mathrm{hrs}$ before the test.

\section{The Density, Pore Size And Porosity}

The density of scaffold was evaluated with the methods described by Fan et al. ${ }^{20}$ Pore diameter was estimated from SEM microphages. The pore size was estimated using a minimum of 30 pores from different places of the crosssection of the scaffolds as decrypted by previous report. ${ }^{21}$ Three different cross-sections of each scaffold were used to estimate pore size. The porosity was measured by the method of liquid displacement as following: the volume $\left(\mathrm{V}_{0}\right)$ and weight $\left(\mathrm{W}_{0}\right)$ of the sample were measured. Then, the sample was immersed into ethanol for 5 mins until it was saturated by ethanol. The sample was weighted again and noted as $\mathrm{W}_{1}$. The porosity of the sample was calculated according to the formula: porosity $(\%)=\left(\mathrm{W}_{1}-\mathrm{W}_{0}\right) /\left(\mathrm{qV}_{0}\right) \times 100$ (q represents the density of the ethanol). The reported data were the average of five samples.

\section{Elasticity Modulus}

Elasticity modulus of the samples was tested with a universal testing machine (ELF 3220 seires2, Bose Co., USA) at room temperature and humidity. The elasticity modulus was calculated as the slope of the initial linear section of the stress-strain curve. The reported data were the average of five samples. 


\section{In Vitro Release Behavior}

The release behavior of salvianolic acid B from Sa B-CS/ HA scaffolds was measured by high-performance liquid chromatography (HPLC, Waters Co., Milford, USA). Sa B-CS/HA scaffolds were soaked in $5 \mathrm{~mL}$ PBS with the $\mathrm{pH}$ 7.4 and shaken gently at the speed of $10 \mathrm{rpm}$. At the time point of 1, 3, 7, 14, 28, 42 and 56 days, the PBS solution was collected and freeze dried into powder, and then $300 \mu \mathrm{L}$ methanol was added into the powder and homogeneously mixed. And, then the solution was filtered by millipore filtration (pore size $=22 \mu \mathrm{m}$ ). The salvianolic acid B standard solutions were made with the concentrations of $5 \mu \mathrm{g} / \mathrm{mL}$, $10 \mu \mathrm{g} / \mathrm{mL}, 20 \mu \mathrm{g} / \mathrm{mL}, 50 \mu \mathrm{g} / \mathrm{mL}$ and $100 \mu \mathrm{g} / \mathrm{mL}$, and measured by HPLC to get the standard concentration-absorbency curve. The concentration of salvianolic acid B released from the scaffold sample at each time point was calculated according to standard curve and the percentage of released salvianolic acid B was accumulated.

\section{In Vitro Bioactivity Cell And Medium Preparation}

MC3T3-E1 cells were purchased from the Cell Bank of the Shanghai Institute of Life Sciences (ATCC CRL-2594, Chinese Academy of Sciences, Shanghai, People's Republic of China) and cultured in DMEM/F12 medium supplemented with 10\% FBS. HUVECs were purchased from the Cell Bank of the Shanghai Institute of Life Sciences (ATCC CRL-1730, Chinese Academy of Sciences, Shanghai, People's Republic of China) and cultured in high glucose DMEM medium supplemented with $10 \%$ FBS. All the cells used in the following experiments were between 5 th and 8 th passage. $1.5 \mathrm{~g}$ sterilized Sal B-CS/ HA scaffolds were cut into discs with $4.0 \mathrm{~mm}$ in diameter and $5 \mathrm{~mm}$ in thickness, then soaked in $15 \mathrm{~mL}$ DMEM/F12 or high glucose DMEM medium at $37^{\circ} \mathrm{C}$ for $72 \mathrm{hrs}$. Then, solid material was removed by millipore filtration (pore size $=22 \mu \mathrm{m})$ and the medium was collected as the conditional cell medium (Sal B-CS/HA group). By the same method, $1.5 \mathrm{~g}$ sterilized $\mathrm{CS} / \mathrm{HA}$ scaffolds without salvianolic acid B were also soaked for $72 \mathrm{hrs}$ and the medium was collected as the normal medium (CS/HA group).

\section{Cell Morphology In Scaffolds}

MC3T3-E1 cells were seeded onto scaffolds with a density of $1 \times 10^{5} / \mathrm{mL}$. After 5 days, samples were fixed by $2.5 \%$ glutaraldehyde and coated with gold. Cell morphology on the surface of scaffold was observed by the method of scanning electron microscopy (SEM).

\section{Cell Proliferation}

The proliferation of MC3T3-E1 cells was evaluated by CCK8 method. MC3T3-E1 cells were seeded in 96-well plate with 2000 cells in each well, and incubated in vitro at $37^{\circ} \mathrm{C}$ in a humidified atmosphere with $5 \% \mathrm{CO}_{2}$. After 1 day, the medium in Sal B-CS/HA group was replaced by $100 \mu \mathrm{L}$ conditional medium, while the CS/HA group changed with normal medium. At 3 and 5 days, $10 \mu \mathrm{L}$ CCK 8 was added into the well and the absorbance (OD) was detected at $450 \mathrm{~nm}$ in a microplate reader (Infinite 200 PRO, TECAN, Switzerland). The proliferation of HUVECs was also evaluated by the same method after 1 and 3 days.

\section{Alkaline Phosphatase (ALP) Activity}

MC3T3-E1 cells were seeded in 6-well plates at a density of $1 \times 10^{6} /$ well. The cells in Sal B-CS/HA group were cultured with the conditioned medium and the cells in CS/HA group were cultured with normal medium. After 7 and 14days, cells were lysed in $500 \mu \mathrm{L}$ deionized water and homogenized by ultrasound at $4^{\circ} \mathrm{C}$. ALP activity and total protein content in cell lysates were measured and quantified using an alkaline phosphatase activity kit (Jiancheng Bioengineering Institute, China) and a microBCA Assay kit (Thermo Inc, USA). ALP activity was normalized for the corresponding total protein concentration $(\mathrm{U} / \mathrm{g})$.

Vascular Endothelial Growth Factor (VEGF) Activity HUVECs were seeded in 6-well plates at a density of $1 \times 10^{6} /$ well, and cells in Sal B-CS/HA group were cultured with conditioned medium and the cells in CS/HA group were cultured with normal medium for 3 days. At 1 day and 3 days after the seeding, the secretory components of medium were detected. The medium was collected with a sterile container and centrifuged at the speed of $3000 \mathrm{r} / \mathrm{m}$ for $20 \mathrm{mins}$. Then, the supernatant was collected and VEGF concentrations in the supernatant were quantified by the VEGF ELISA assay kit (RD Biosciences Inc, USA).

\section{In Vivo Tests}

\section{Animal Grouping And Surgery}

All experiments were in compliance with the Guide for the Care and Use of Laboratory Animals of the National Institutes of Health. The protocol was approved by the Animal Care and Experimental Ethics Committee of Air Force Medical University. All efforts were made to minimize animal suffering and sacrifice. 
Forty rabbits were randomly divided into two groups with 20 rabbits in each group. For all rabbits, a longitudinal incision was made at the radius of the anterior limb to expose the radius under general anesthesia (Figure 1A). Then, a radius osteotomy of $1.5 \mathrm{~cm}$ length was performed in the middle shaft of the radius and the ulna was retained to avoid the fracture of limb (Figure 1B). The Sa B-CS/HA scaffolds or CS/HA scaffolds were imbedded into the defect sites (Figure 1C). All incisions were closed using nonabsorbable sutures, and $400,000 \mathrm{U}$ of penicillin was administrated daily by intramuscular injection for 3 days (Figure 1D).

\section{CT-Based Bone Analysis}

The implanted limbs of animals were scanned by CT machine in cross-sectional manner at 6 and 12 weeks. The three-dimensional reconstruction was performed with a standardize segmentation parameter. The three-dimensional reconstructive images of samples were generated by machine built-in software to assess osseous tissue fusion between two processes.

\section{Histology And Immunohistochemistry}

Five animals of each group were collected randomly and sacrificed to collect the bone graft at 6 and 12 weeks.

\section{CS/HA}

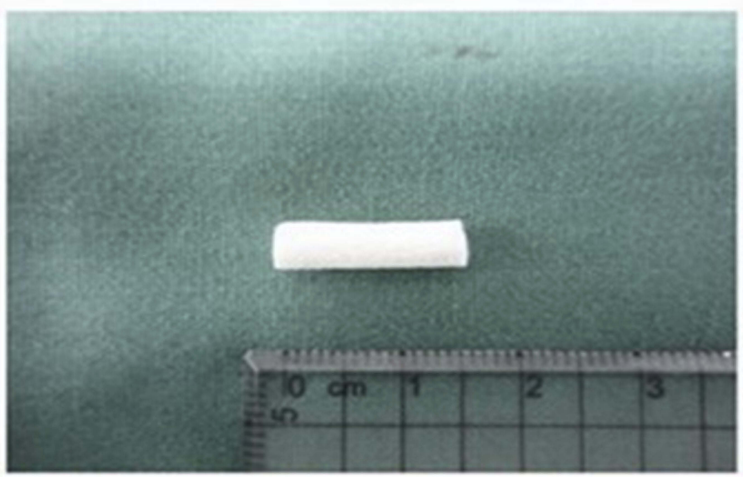

HE

SEM
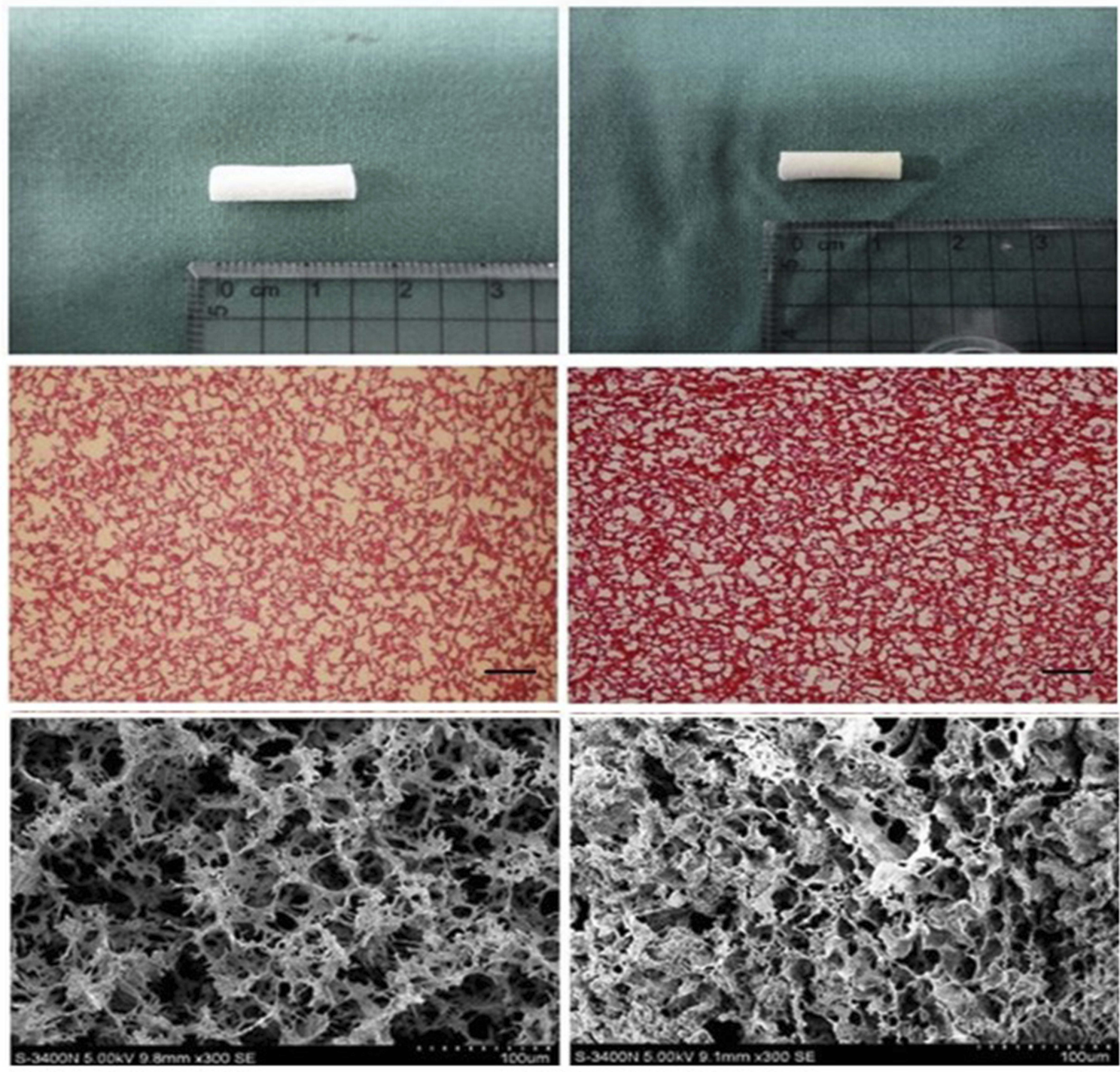

\section{Sa B-CS/HA}

Figure I HE staining and SEM micrographs of CS/HA (left) and Sa B-CS/HA (right).

Notes: $\mathrm{HE} \times 40$, bar $250 \mu \mathrm{m}$; SEM $\times 300$, bar $100 \mu \mathrm{m}$. 
Specimens were fixed in $4 \%$ buffered paraformaldehyde, decalcified in $50 \mathrm{mM}$ ethylene diaminetetraacetic acid (EDTA), serially dehydrated, infiltrated in isoamyl alcohol, embedded in paraffin, and sectioned into $4-\mu \mathrm{m}$ thickness. Twenty sections were made for each sample with 10 being for staining with haematoxylin and eosin (HE). The degree of bone regeneration was calculated as percent area of new bone within the bone graft and measured using the image analysis software (Image-Pro Plus 6.0, USA). The mean percentage of new bone area was calculated and compared between groups. The other 10 sections of each sample were used for immunostaining. The sections were then incubated in one of the primary antibodies (anti-CD34 1:100, Wuhan Boster Biological Technology Ltd., China) for $2 \mathrm{hrs}$ and incubated in second antibodies for 20 mins at room temperature. Then, they were further processed using SABC immunostaining kit (Wuhan Boster Biological Technology Ltd.) according to the manufacturer's instruction. Known CD34 expression slides were used as positive controls and PBS was replaced with primary antibodies as negative control. The number of new blood vessels at the implantation site was determined by analyzing the sections immunostained with anti-CD34 antibodies and the total number of vessels on complete implant sections was recorded with Image-Pro Plus software.

\section{Statistical Analysis}

Statistical analyses were performed using SPSS software, version 12.0 (SPSS Inc, USA). The data were presented as the mean \pm standard deviation. Statistical analysis between two samples was compared by Student's $t$-test. $\mathrm{P}<0.05$ was considered statistically significant.

\section{Results And Discussion Characterization Of Scaffolds}

According to the results of HE staining and SEM, CS/HA scaffold had interconnected porous structure and the diameter of the pores was about $100-300 \mu \mathrm{m}$. The pore size of Sa B-CS/HA scaffold was similar at $85-290 \mu \mathrm{m}$. Similar porous structure was observed for both scaffolds, implying that salvianolic acid B loading procedure did not obviously alter the microstructure of the scaffold (Figure 2).

FTIR spectra of pure salvianolic acid $\mathrm{B}, \mathrm{CS} / \mathrm{HA}$ and $\mathrm{Sa}$ B-CS/HA were compared. The characteristic peaks of salvianolic acid $B$ appeared at 1680 and $1560 \mathrm{~cm}^{-1}$. The characteristic peaks of CS/HA were at $3390 \mathrm{~cm}^{-1}, 1650 \mathrm{~cm}^{-1}$ and 1400 $\mathrm{cm}^{-1}$. When salvianolic acid B was loaded into the CS/HA, all the characteristic peaks of salvianolic acid B were still appeared in the curve at 1680 and $1560 \mathrm{~cm}^{-1}$, and the characteristic peaks of CS/HA were also still appeared. So, the salvianolic acid B did not get any chemical change when it was loaded onto CS/HA (Figure 3).

The porosity of CS/HA scaffold without loading salvianolic acid B was $86.3 \pm 1.2 \%$, and the density was $86.4 \pm 1.2 \mathrm{~kg} / \mathrm{m}^{3}$. After loading the drug, the porosity of the Sa B-CS/HA scaffold was decreased to $83.3 \pm 0.8 \mathrm{~kg} / \mathrm{m}^{3}$ and the density was increased to $94.2 \pm 1.6 \mathrm{~kg} / \mathrm{m}^{3}$. The elastic modulus was also decreased from $34.3 \pm 3.7 \mathrm{kP}$ to $25.2 \pm 2.9 \mathrm{kP}$ after loading the drug (Table 1).

\section{In Vitro Controlled Release Behavior}

The release behavior of Sa B-CS/HA scaffold was investigated by HPLC examination. The concentration of salvianolic acid B was calculated based on standard curve and demonstrated as the accumulated percentage. As our result showed the release of salvianolic acid B was durable for more than 56 days and about 35\% drug released from the scaffold after 56 days. And, the release of salvianolic acid B at the early phase from day 1 to day 14 was relatively rapid with approximately $10 \%$ to $22 \%$ drugs released. From day 28 to day 56, the release of salvianolic acid B was slow down and maintained steady with approximately $30 \%$ to $35 \%$ drug released (Figure 4 ).

\section{In Vitro Bioactivity}

The morphology of MC3T3-E1 cells on the scaffold is shown in Figure 5. There were no obvious differences of cell morphology between the CS/HA and Sa B-CS/HA group. The cells on both kinds of scaffolds spread very well and stretched out pseudopods to closely hold on the surfaces of scaffolds or others cells after 5 days culture.

The proliferation of MC3T3-E1 cells cultured in the Sa B-CS/HA conditional medium was promoted compared with the control group at 3 and 5 days. The differences were significant at each time point between two groups $(\mathrm{P}<0.05)$ (Figure 6). The proliferation of HUVECs was also promoted in the conditional medium compared with the control group at 1 and 3 days. The differences were significant at each time point between two groups $(\mathrm{P}<0.05)$ (Figure 7).

ALP activity expressed by the MC3T3-E1 cells after 7 and 14days of culture is shown in Figure 8. ALP activity of the cells in Sa B-CS/HA group cultured with the 


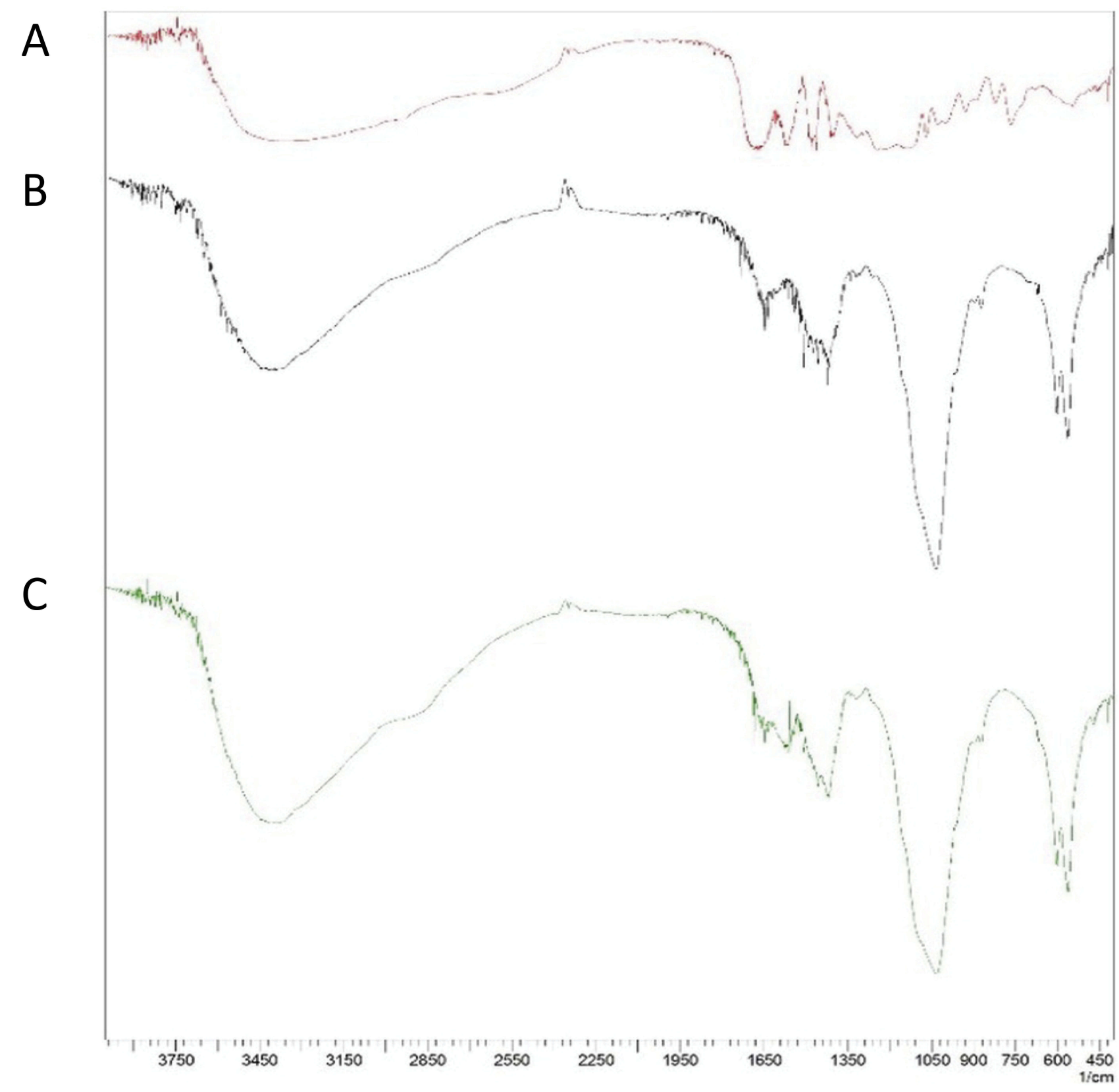

Figure 2 FTIR spectra of pure salvianolic acid B (A), CS/HA (B) and Sa B-CS/HA (C).

conditional medium was significantly higher than in CS/ HA group at each time point $(\mathrm{P}<0.05)$.

VEGF activity secreted by HUVECs after 1 and 3 days of culture is shown in Figure 9. VEGF of the cells in Sa B$\mathrm{CS} / \mathrm{HA}$ group cultured with the conditional medium was significantly higher than in the CS/HA group at day 3 $(\mathrm{P}<0.05)$.

\section{In Vivo Tests}

CT examinations were performed to evaluate the development of bone regeneration within the defects (Figure 10).
After 6 weeks in Groups CS/HA, the area of bone defect was still visually observed without obvious bone formation. While the bony callus began to be absorbed in Group Sa BCS/HA with less bone defect area indicating better bony formation. After 12 weeks, Group Sa B-CS/HA exhibited significant bone formation and most of the defect area was filled with whole bone cortex while the bone defect was still obvious in Group CS/HA (Figure 10A). After 6 weeks, results show that bone volume ratio (BV/TV) was significantly higher in Group Sa B-CS/HA compared with control group $(29.6 \%$ vs $16.2 \%$ in BV/TV, p $<0.05$, Figure $10 \mathrm{~B})$. 


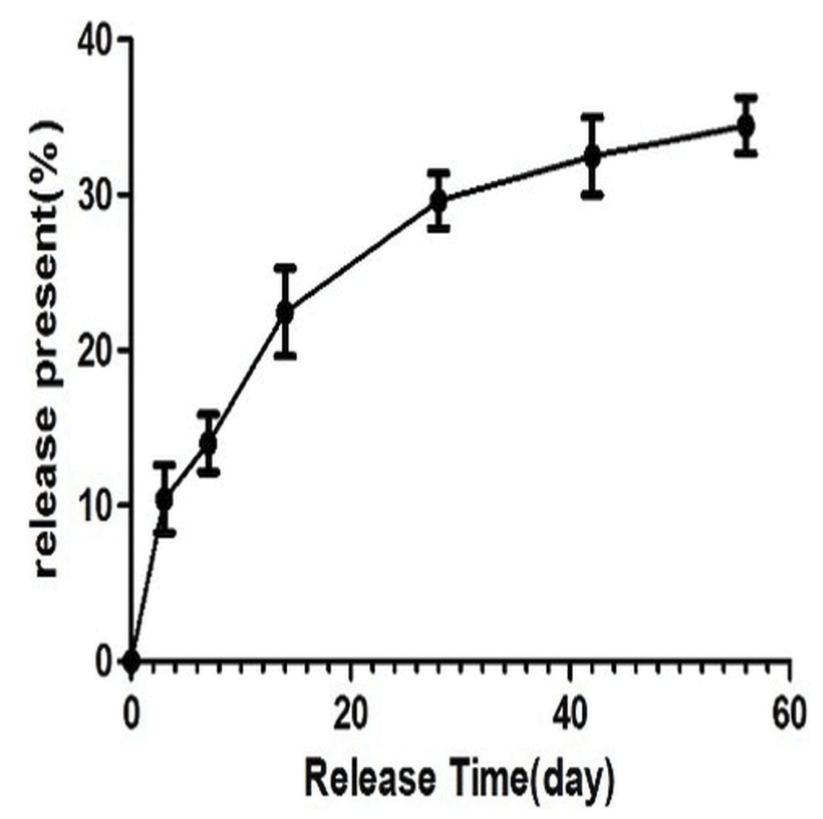

Figure 3 Release behavior of salvianolic acid B from Sa B-CS/HA scaffold in vitro for 8 weeks.

After 12 weeks, although new forming bone was increased in Groups $\mathrm{CS} / \mathrm{HA}$, it was still much lower than the bone volume in Group Sa B-CS/HA (45.5\% vs $74.4 \%$ in BV/TV, $\mathrm{p}<0.05$, Figure 10B).

As shown in Figure 11A, callus was obvious in the specimens at 6 weeks in both Groups. There was a difference between the two groups with more callus masses absorbed in Group Sa B-CS/HA. After 12 weeks, although bone cortex began to form in Group CS/HA, the process proceeded slowly. Group Sa B-CS/HA led to a better result with complete bone cortex regeneration. After 6 and 12 weeks, the percentage of bone formation in the initial defect site in Group CS/HA was higher than in Group Sa B-CS/HA. These results are summarized in Figure 11 and the percentage of bone formation in the grafts was much higher in Group Sa B-CS/HA (p < 0.05) (Figure 11B), which was consistent with the $3 \mathrm{D}$ results of $\mathrm{CT}$ analysis.

As shown in Figure 12A, CD34 was found expressed in vascular endothelial cell, with significant increases in

Table I Morphology And Mechanical Properties

\begin{tabular}{|l|l|l|l|l|}
\hline Group & $\mathbf{N}$ & $\begin{array}{l}\text { Density } \\
\left(\mathbf{k g} / \mathbf{m}^{\mathbf{3}}\right)\end{array}$ & $\begin{array}{l}\text { Porosity } \\
\mathbf{( \% )}\end{array}$ & $\begin{array}{l}\text { Elastic Modulus } \\
\mathbf{( k P a )}\end{array}$ \\
\hline CS/HA & 5 & $86.4 \pm 1.2$ & $86.3 \pm 1.2$ & $34.3 \pm 3.7$ \\
Sa B-CS/HA & 5 & $94.2 \pm 1.6^{*}$ & $83.3 \pm 0.8^{*}$ & $25.2 \pm 2.9^{*}$ \\
\hline
\end{tabular}

Note: ${ }^{*} \mathrm{P}<0.05$. both the size and area of vessels in Group Sa B-CS/HA compared with Group CS/HA ( $<$ 0.05) (Figure 12B).

\section{Discussion}

The implantation of biomaterials which can efficiently release bioactive agents at the location of bone defect is regarded as a good method to repair large bone defect. ${ }^{2}$ Among these bioactive agents, growth factors such as bone morphogenetic proteins (BMPs) and vascular endothelial growth factor (VEGF) have been extensively studied. ${ }^{22,23}$ However, the high cost and rapid degradation limit their widespread use in the clinic. Besides, the effect of the osteogenesis is influenced by the situation of angiogenesis. $^{24,25}$ So, there is an urgent to develop some alternative agents with both osteogenesis and angiogenesis to get better effect of bone formation. Salvianolic acid B is a drug that has been proven to have the bioactivities of both angiogenesis and osteogenesis. ${ }^{7,10}$ So, we chose this drug in our experiment to be loaded in the bone scaffold. As our result showed the osteoblast cultured in the conditional medium with salvianolic acid B had better cell proliferation and alkaline phosphatase activity compared with the normal medium. The HUVECs cultured in the conditional medium with salvianolic acid $\mathrm{B}$ also had better cell proliferation and vascular endothelial growth factor activity compared with the normal medium. Our results were consistent with the previous reports and further proved the good bioactivities of angiogenesis and osteogenesis of the salvianolic acid B in vitro.

To be used as an ideal bone scaffold, the drug-loaded scaffolds for drug delivery should have good release behavior. ${ }^{13}$ They should control the drug release and maintain the local drug concentration steady and durable. The drug loading procedure should not significantly affect the characterization of the scaffold. As our result showed the release of salvianolic acid B was durable for more than 56 days in vitro. The release rate of salvianolic acid B was fast at the beginning 30 days with almost 30\% drug release and then the release rate was slow down with less than $10 \%$ drug release for next 26 days. In our previous study, the CS/HA scaffold was proven to be a feasible drugloaded system to load icariin. ${ }^{20}$ And by the same method, this drug-loaded system was further proved to be feasible to load the salvianolic acid B.

After loading the salvianolic acid B, our result showed that salvianolic acid B got no obvious chemical change when it was loaded onto CS/HA and the microstructure of the scaffold was not significantly changed. After loading 


\section{$\mathrm{CS} / \mathrm{HA}$}

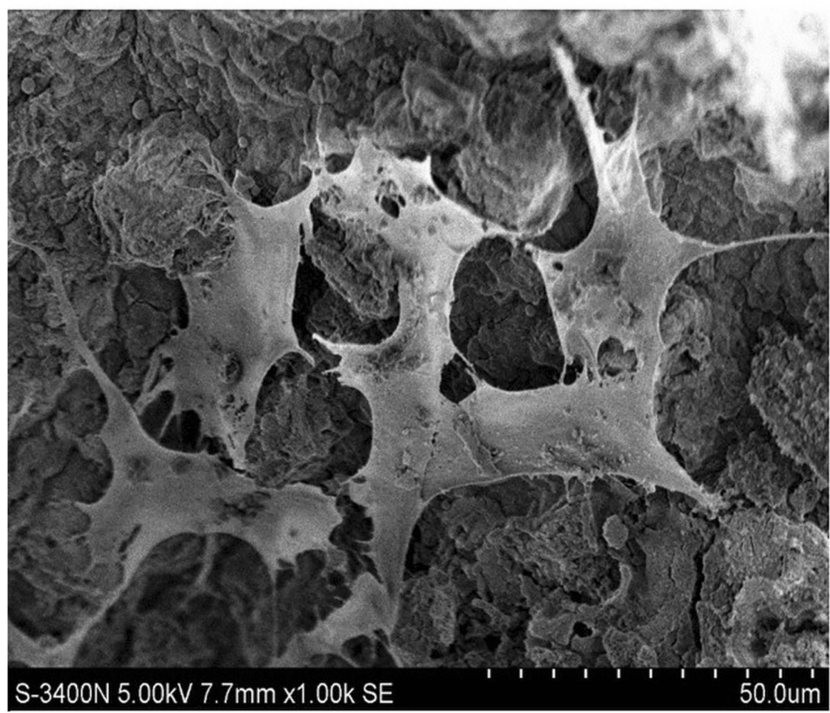

\section{Sa B-CS/HA}

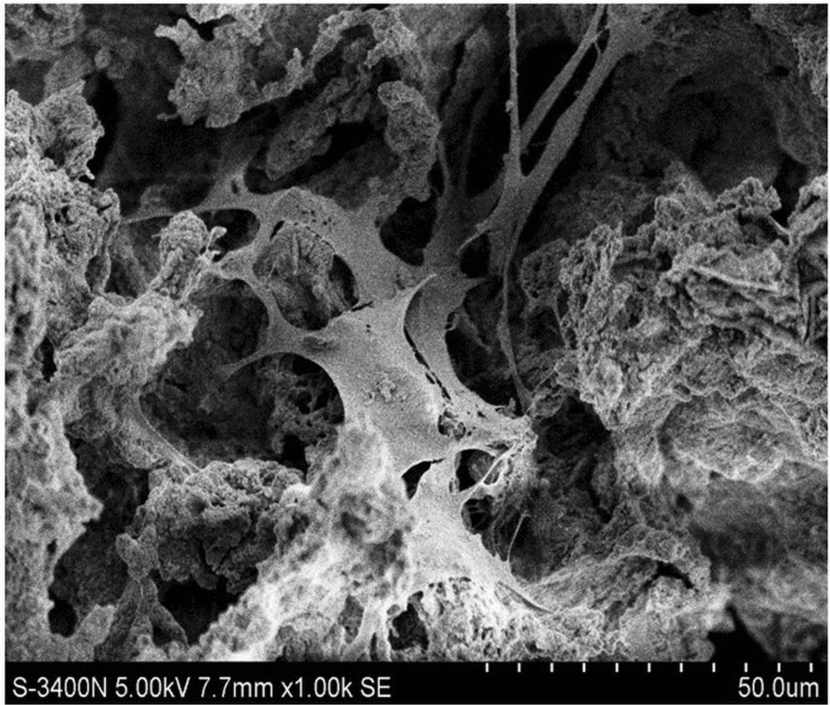

Figure 4 Surface morphology of MC3T3-EI cells cultured in CS/HA (left) and Sa B-CS/HA scaffolds (right).

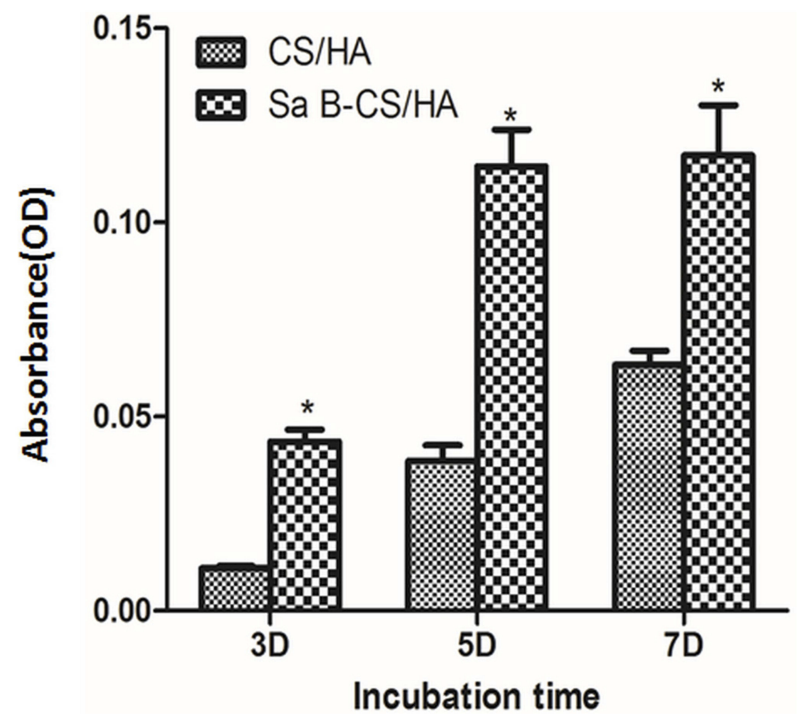

Figure 5 Proliferation of MC3T3-EI cells in CS/HA and Sa B-CS/HA groups at 3 and 5 days.

Notes: Values represent the mean of 3 times in each group (mean \pm SD). *Significant difference between the values of Sa $\mathrm{B}-\mathrm{CS} / \mathrm{HA}$ group and $\mathrm{CS} / \mathrm{HA}$ group $(\mathrm{P}<0.05)$.

the salvianolic acid B, the porosity of scaffold was slightly decreased, and the density of the scaffolds was slightly increased. The elastic modulus was also affected after loading the drug. Although different stiffness of material has an influence on the differentiation of stem cells, this mild change of elastic modulus was acceptable and still under the range of suitable stiffness which could induce the osteogenic differentiation. ${ }^{26}$ And, these mild changes of the scaffold after drug loading would not significantly

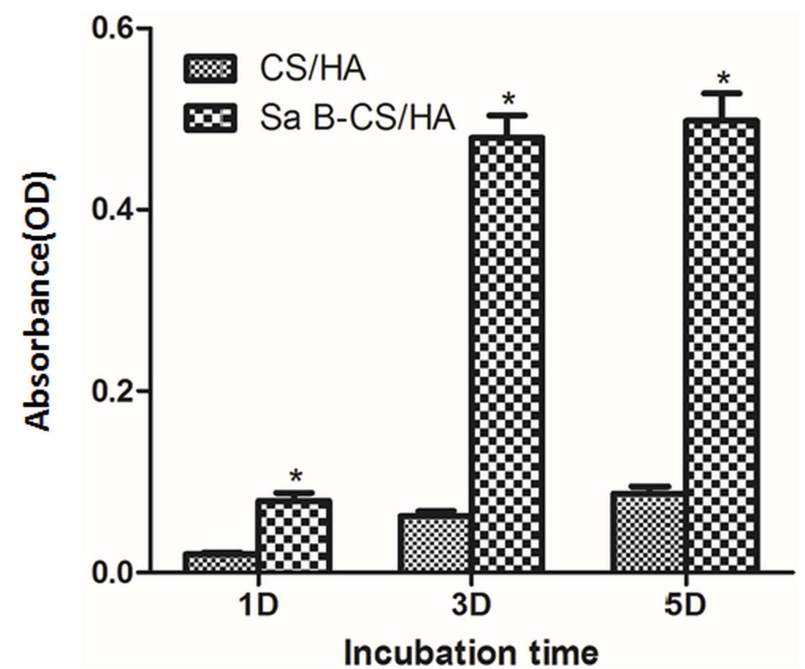

Figure 6 Proliferation of HUVEC in CS/HA and Sa B-CS/HA groups at I and 3 days..

Notes: Values represent the mean of 3 times in each group (mean $\pm S D$ ). *Significant difference between the values of Sa B-CS/HA group and CS/HA group $(P<0.05)$.

influence their use in non-weight-bearing area as a bone repair scaffold.

Besides controlled drug release behavior, the bone scaffold should also have good biocompatibility and bioactivity. ${ }^{13}$ As our result showed the morphology of MC3T3-E1 cells on the Sa B-CS/HA scaffold was good and not obviously different from that of CS/HA scaffold. For the study of in vitro bioactivity, we used the extract of the scaffolds to get the conditional medium. And, the 


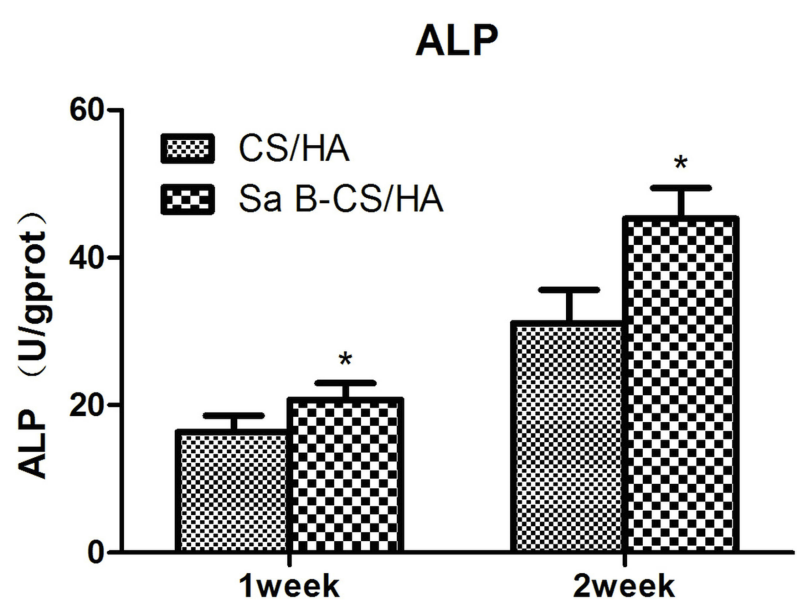

Figure 7 ALP activity of MC3T3-EI cells cultured in CS/HA and Sa B-CS/HA groups at 7 and 14 days.

Notes: Values represent the mean of 3 times in each group (mean $\pm S D)$. *Significant difference between the values of Sa $\mathrm{B}-\mathrm{CS} / \mathrm{HA}$ group and $\mathrm{CS} / \mathrm{HA}$ group $(\mathrm{P}<0.05)$.

\section{VEGF}

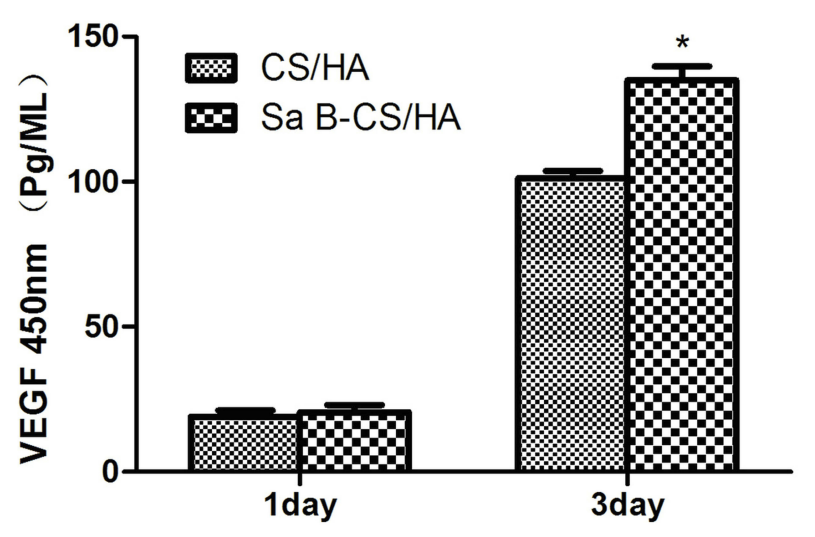

Figure 8 VEGF activity of HUVEC cultured in CS/HA and Sa B-CS/HA groups at I and 3 days..

Notes: Values represent the mean of 3 times in each group (mean $\pm S D$ ). *Significant difference between the values of Sa B-CS/HA group and CS/HA group $(P<0.05)$. conditional medium extracted from the Sa B-CS/HA scaffold could promote the cell proliferation of both MC3T3E1 and HUVECs significantly. The alkaline phosphatase activity and vascular endothelial growth factor activity were also increased in the Sa B-CS/HA group compared with the CS/HA group. The biocompatibility and bioactivity of this Sa B-CS/HA were proven to be favorable by our in vitro results.

Our in vivo experiments further demonstrated the good efficacy of Sa B-CS/HA scaffold for bone defect repairing. Both CT and histological evaluation suggested that the $\mathrm{Sa}$ B-CS/HA scaffold can enhance bone regeneration. The formation of new cortex was observed via inspection of Group Sa B-CS/HA (6 and 12 weeks). The formation of the new bone was increased as shown by CT and histology analysis with the time and the Group Sa B-CS/HA had better osteogenesis effect. Further analysis using the CD34 immunostaining indicated that $\mathrm{Sa} \mathrm{B}-\mathrm{CS} / \mathrm{HA}$ scaffold also had better angiogenesis in vivo. Our results were similar to other reports about Sa B-loaded scaffold used for bone regeneration. ${ }^{27,28}$ And, there evidences proved that the $\mathrm{Sa}$ B-loaded scaffold had the good efficacy for bone defect repairing with both angiogenesis and osteogenesis effect.

However, there are some other limitations in our study. Despite Sa B-CS/HA scaffold had significant effect on bone formation compared with that of pure CS/HA scaffold, but no blank group or autograft group was used for comparison. Actually, the $1.5 \mathrm{~cm}$ length of radius bone defect in rabbit was the acknowledged critical defect size that could not self-repair, ${ }^{29}$ so the blank group was not used in our study. And, autograft was still the golden standard for bone defect repair, however, we just used the pure CS/HA scaffold without loading drug as the

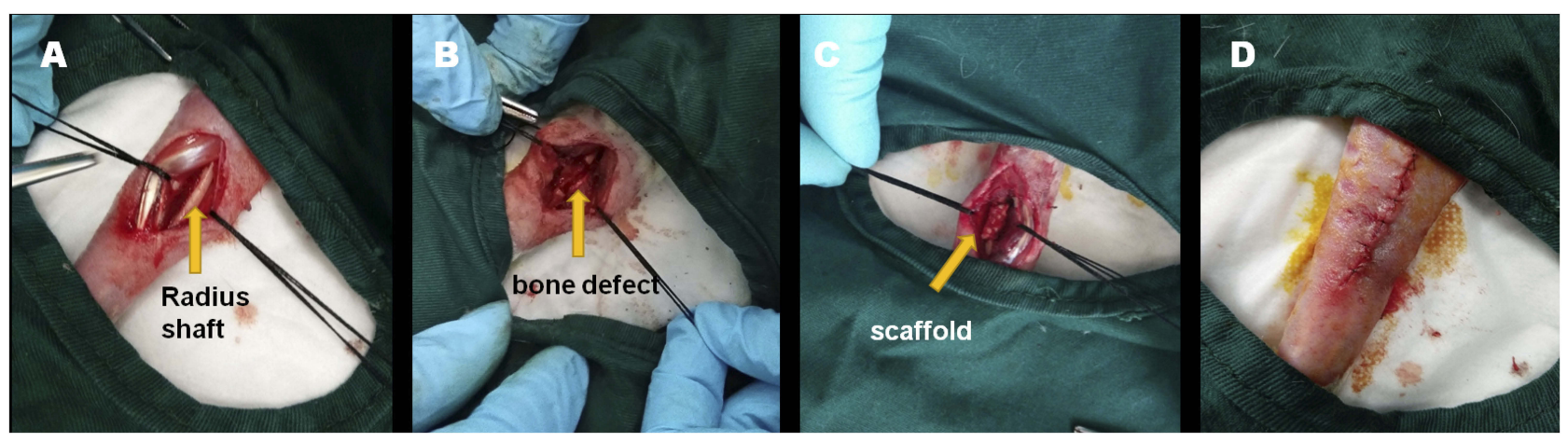

Figure 9 Surgery process of the rabbit model.

Notes: (A) Longitudinal incision was made at the radius of the anterior limb to expose the radius under general anesthesia. (B) A radius osteotomy of $\mathrm{I.5} \mathrm{cm}$ length was performed in the middle shaft of the radius and the ulna was retained to avoid the fracture of limb. (C) The Sa B-CS/HA scaffolds or CS/HA scaffolds were imbedded into the defect site. (D) All incisions were closed using nonabsorbable sutures. 
A

CS/HA

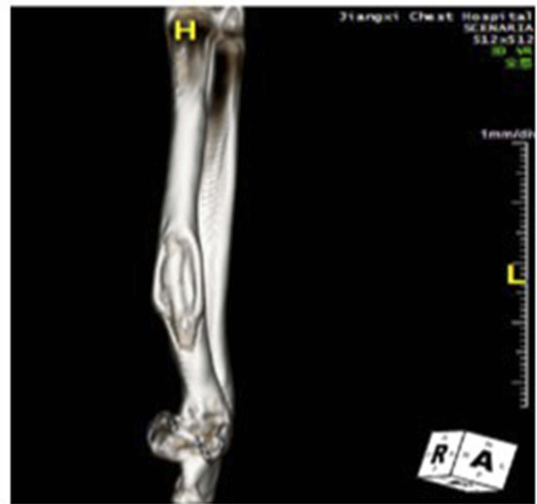

$6 \mathrm{~W}$

$12 \mathrm{~W}$
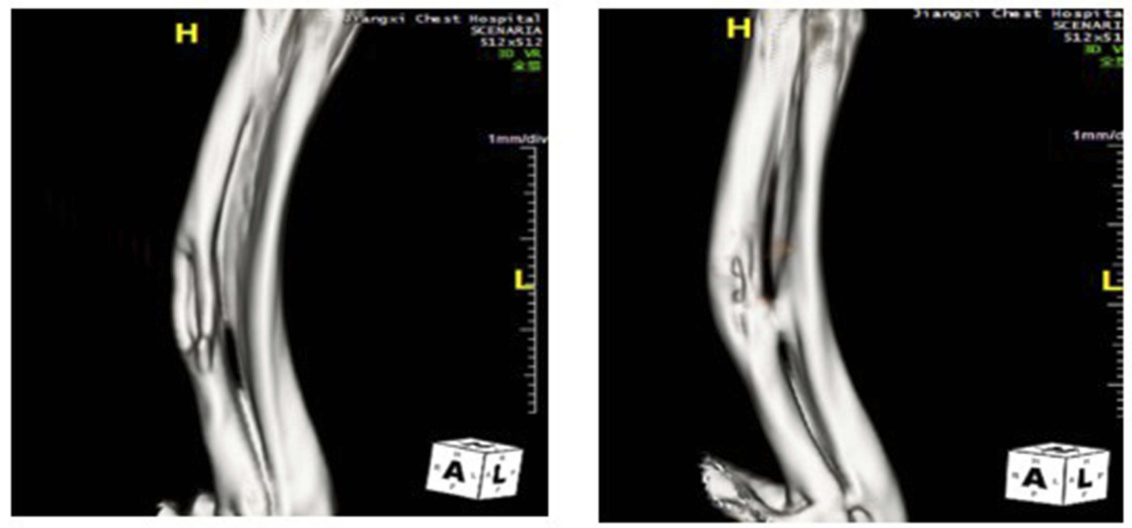

B

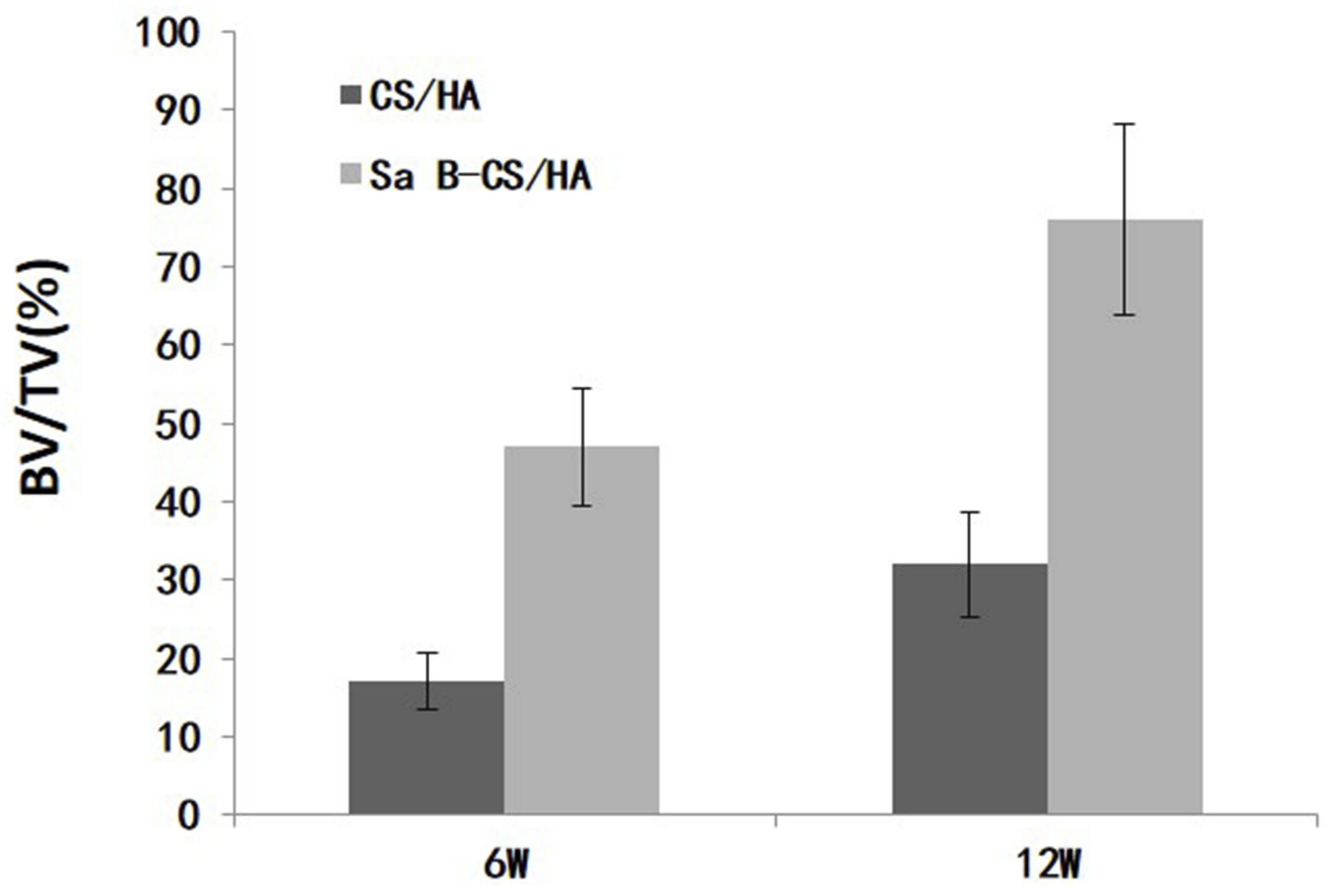

Figure 10 CT images and analysis of new bone formation.

Notes: (A) After 6 weeks and 12 weeks, Group Sa B-CS/HA exhibited significant bone formation and most of the defect area was filled with whole bone cortex while the bone defect was still obvious in Group CS/HA. (B) Bone volume ratio (BV/TV) was significantly higher in Group Sa B-CS/HA compared with control group $(p<0.05)$. 
A

6W

$12 \mathrm{~W}$

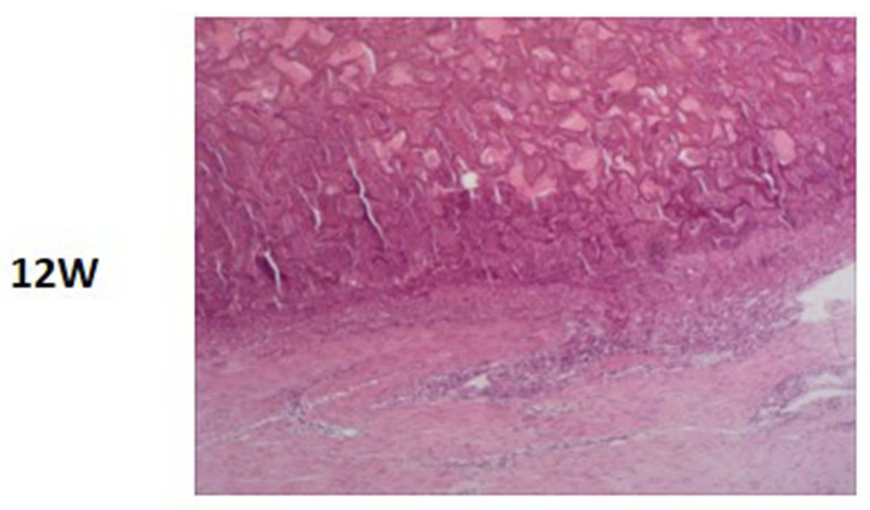

CS/HA

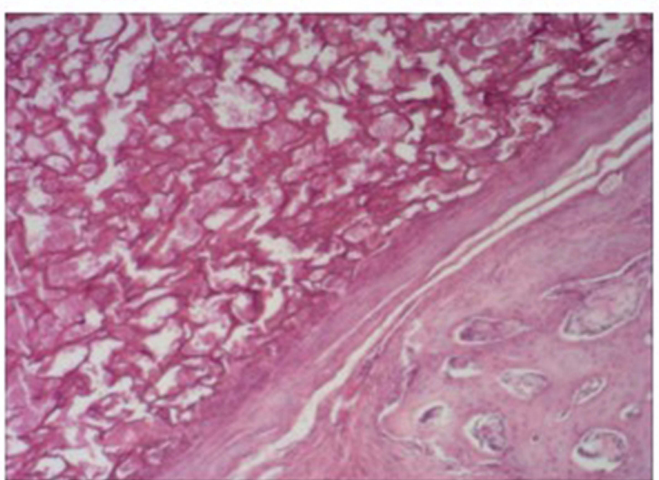

Sa B-CS/HA
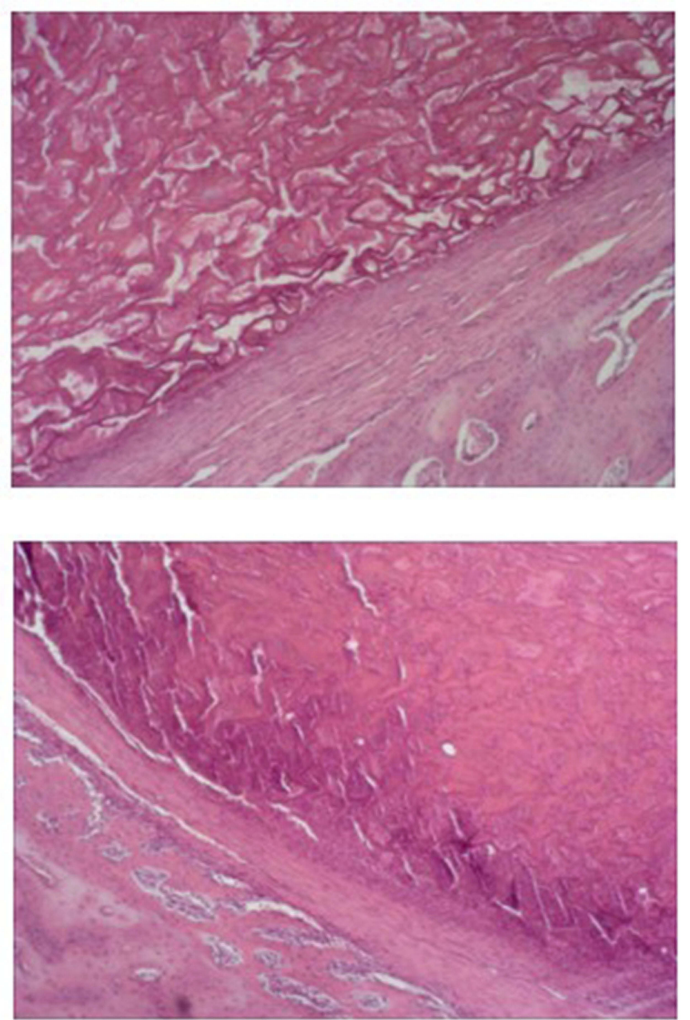

B

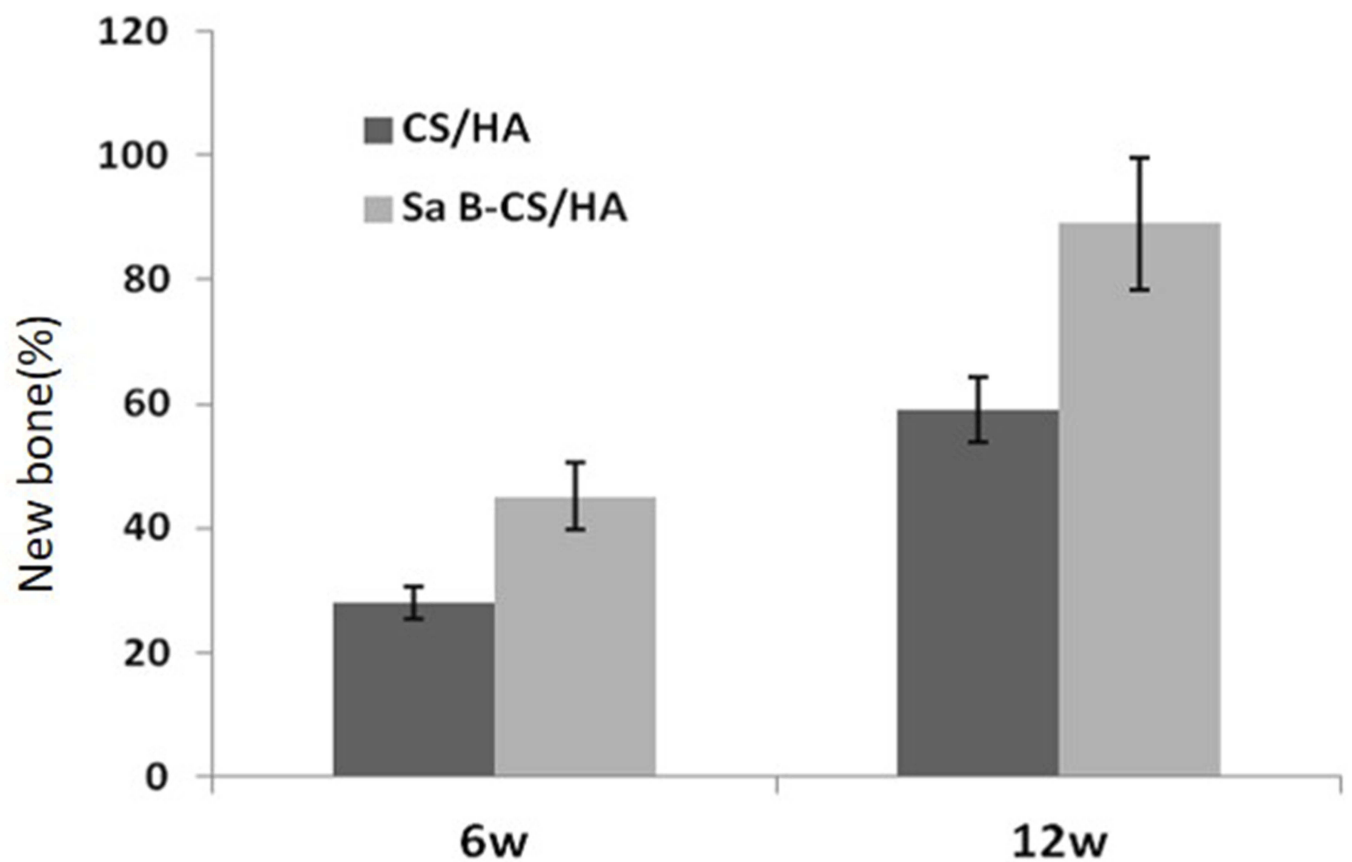

Figure I I Histological images and histomorphometric analysis of bone formation.

Notes: (A) Histological micrographs of sections upon HE staining. After 6 and 12 weeks, although bone cortex began to form in Group CS/HA, the process proceeded slowly. Group Sa B-CS/HA led to a better result with complete bone cortex regeneration. (B) Histomorphometric analysis. After 6 weeks, the percentage of bone formation in the initial defect site in Group CS/HA was higher than in Group Sa B-CS/HA. the percentage of bone formation was significantly higher in Group Sa B-CS/HA compared with Group CS/HA (p<0.05). 
A

6W

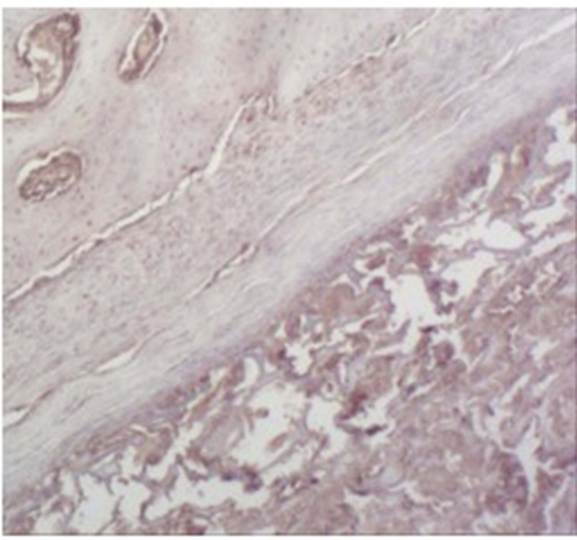

$12 W$

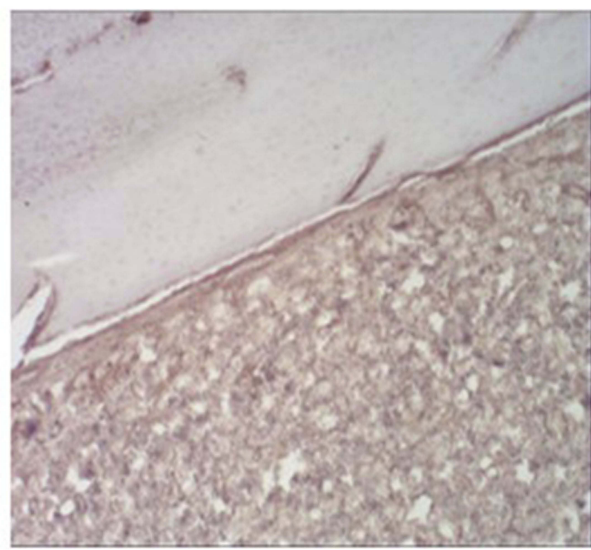

\section{Sa B-CS/HA}
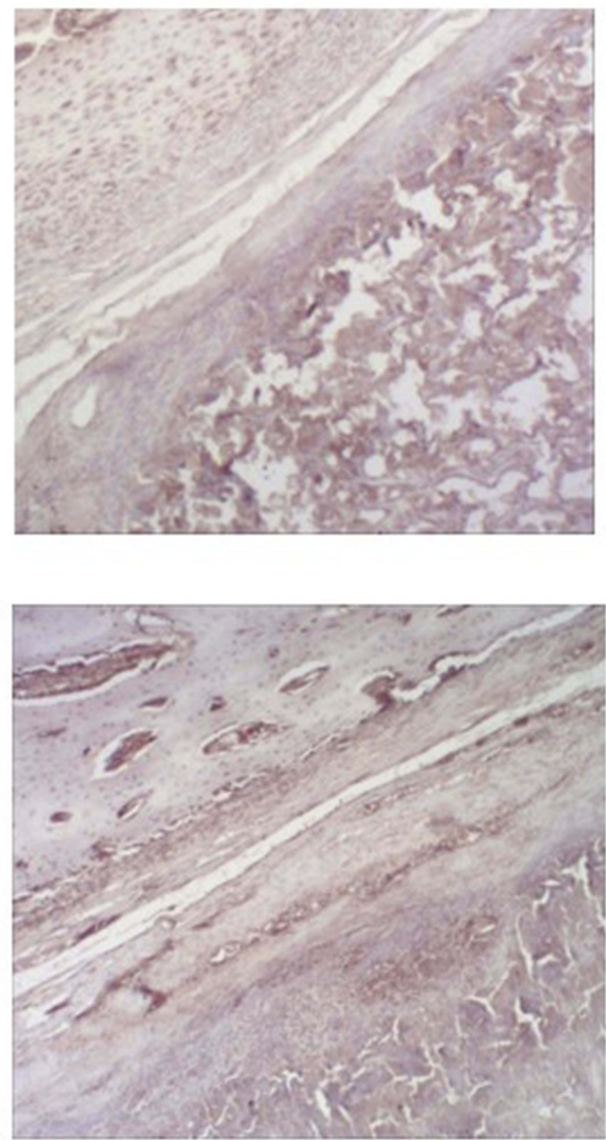

B

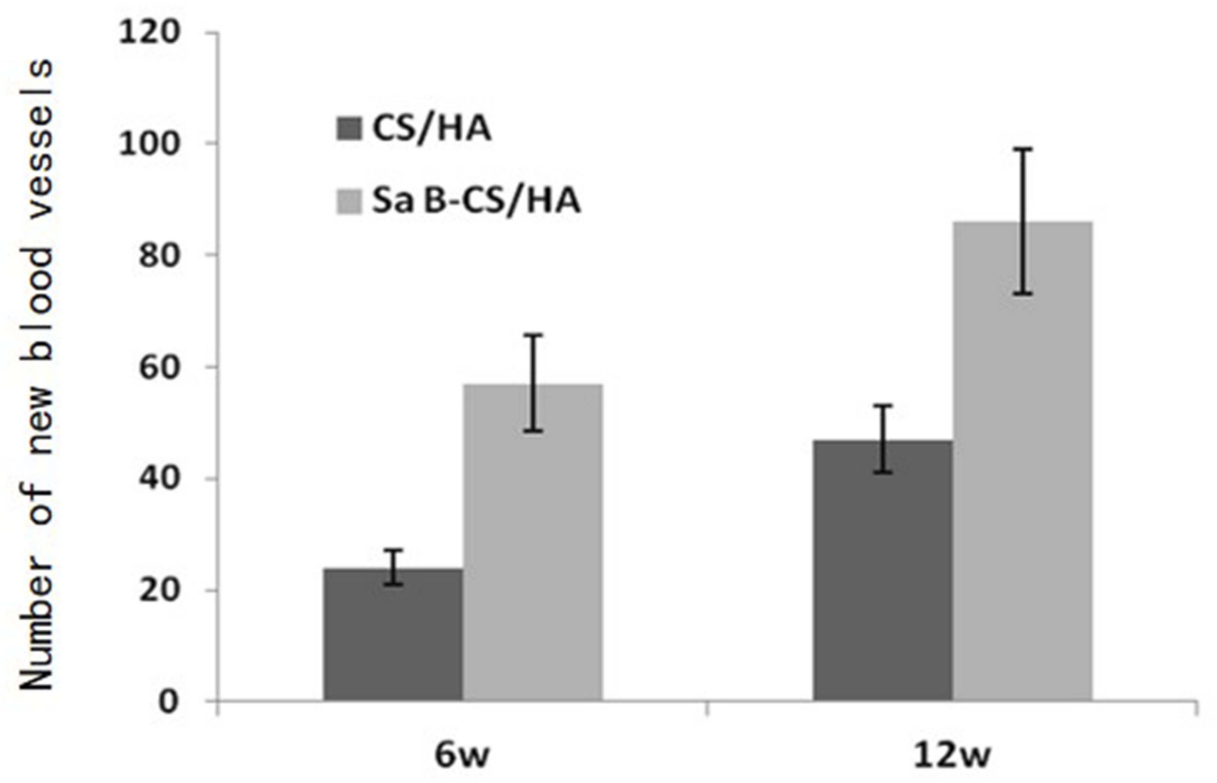

Figure I 2 Results of immunohistochemistry conducted at decalcified samples.

Notes: (A) Representative images of immunohistochemical staining of CD34. (B) CD34 was found expressed in vascular endothelial cell, with significant increases in both the size and area of vessels in Group Sa B-CS/HA compared with Group CS/HA $(p<0.05)$. 
control group. In our next study, we will add autograft group as the positive group to study more details. Second, further study still needs to be tested if higher or lower concentration of Sa B incorporation had better angiogenesis and osteogenesis effect. Third, further study on larger animal such as sheep or pig is necessary to validate the findings of the present study. Lastly, the scaffold materials tested in this study did not degrade completely in week 12 . The presence of residual scaffold materials might interfere with the measurement of new bone formation at week 6 and 12, and a longer study duration still needs further study. Nevertheless, our results shed light onto the loading of Sa B as effect agent to current bone scaffold with the angiogenesis and osteogenesis effect.

\section{Conclusions}

Our study showed that this Sa B-CS/HA scaffold was a promising bone scaffold which could release the salvianolic acid $\mathrm{B}$ durably and steadily. The properties of salvianolic acid $\mathrm{B}$ and $\mathrm{CS} / \mathrm{HA}$ were not significantly changed in the scaffold developed during the production. The drug release from this $\mathrm{Sa} \mathrm{B}-\mathrm{CS} / \mathrm{HA}$ scaffold could last for more than 56 days. The osteogenic and angiogenic bioactivities of the Sal B-CS/HA scaffold were proved to be effective by in vivo and in vitro tests. In summary, our results suggested that this salvianolic acid B-loaded bone scaffold has the potential to be used for bone defect repair with better osteogenic and angiogenic bioactivities.

\section{Acknowledgments}

This work was supported by the National Natural Science Foundation of China (81672189).

\section{Disclosure}

The authors report no conflicts of interest in this work.

\section{References}

1. Kretlow JD, Mikos AG. Review: mineralization of synthetic polymer scaffolds for bone tissue engineering. Tissue Eng. 2007;13:927-938 doi:10.1089/ten.2006.0394

2. Kazemzadeh-Narbat M, Noordin S, Masri BA, et al. Drug release and bone growth studies of antimicrobial peptide-loaded calcium phosphate coating on titanium. J Biomed Mater Res B Appl Biomater. 2012;100:1344-1352. doi:10.1002/jbm.b.32701

3. Hong MH, Son JS, Kim KM, et al. Drug-loaded porous spherical hydroxyapatite granules for bone regeneration. J Mater Sci Mater Med. 2011;22:349-355. doi:10.1007/s10856-010-4197-z

4. Lovett M, Lee K, Edwards A, Kaplan DL. Vascularization strategies for tissue engineering. Tissue Eng Part B Rev. 2009;15:353-370. doi:10.1089/ten.TEB.2009.0085
5. Phelps EA, Garcia AJ. Engineering more than a cell: vascularization strategies in tissue engineering. Curr Opin Biotechnol. 2010;21:704709. doi:10.1016/j.copbio.2010.06.005

6. Tang Y, Jacobi A, Vater C, Zou X, Stiehler M. Salvianolic acid B protects human endothelial progenitor cells against oxidative stress-mediated dysfunction by modulating Akt/mTOR/4EBP1, p38 MAPK/ATF2, and ERK1/2 signaling pathways. Biochem Pharmacol. 2014;90:34-49. doi:10.1016/j.bcp.2014.04.008

7. Lay IS, Chiu JH, Shiao MS, Lui W-Y, Wu C-W. Crude extract of salvia miltiorrhiza and salvianolic acid B enhance in vitro angiogenesis in murine SVR endothelial cell line. Planta Med. 2003;69:26-32. doi: $10.1055 / \mathrm{s}-2003-37034$

8. Guo HD, Cui GH, Tian JX, et al. Transplantation of salvianolic acid B pretreated mesenchymal stem cells improves cardiac function in rats with myocardial infarction through angiogenesis and paracrine mechanisms. Int J Cardiol. 2014. doi:10.1016/j.ijcard.2014.08.104

9. Liu YR, Qu SX, Maitz MF, Tan R, Weng J. The effect of the major components of salvia miltiorrhiza bunge on bone marrow cells. $J$ Ethnopharmacol. 2007;111:573-583. doi:10.1016/j.jep.2007.01.005

10. Cui L, Li T, Liu Y, et al. Salvianolic acid B prevents bone loss in prednisone-treated rats through stimulation of osteogenesis and bone marrow angiogenesis. PLoS One. 2012;7:e34647. doi:10.1371/journal.pone. 0034647

11. Xu D, Xu L, Zhou C, et al. Salvianolic acid B promotes osteogenesis of human mesenchymal stem cells through activating ERK signaling pathway. Int J Biochem Cell Biol. 2014;51:1-9. doi:10.1016/j.biocel. 2014.03.005

12. Amini AR, Laurencin CT, Nukavarapu SP. Bone tissue engineering: recent advances and challenges. Crit Rev Biomed Eng. 2012;40:363408.

13. Fernandez-Yague MA, Abbah SA, McNamara L, et al. Biomimetic approaches in bone tissue engineering: integrating biological and physicomechanical strategies. Adv Drug Deliv Rev. 2015;84:1-29.

14. Shuai C, Gao C, Feng P, Peng S, Wen X. Grain growth associates mechanical properties in nano-hydroxyapatite bone scaffolds. $J$ Nanosci Nanotechnol. 2013;13:5340-5345. doi:10.1166/jnn.2013.7564

15. Sulaiman SB, Keong TK, Cheng $\mathrm{CH}$, et al. Tricalcium phosphate/ hydroxyapatite (TCP-HA) bone scaffold as potential candidate for the formation of tissue engineered bone. Indian J Med Res. 2013; 137:1093-1101.

16. Ma XY, Feng YF, Ma ZS, et al. The promotion of osteointegration under diabetic conditions using chitosan/hydroxyapatite composite coating on porous titanium surfaces. Biomaterials. 2014;35:72597270. doi:10.1016/j.biomaterials.2014.05.028

17. He X, Liu Y, Yuan X, Lu L, Agarwal S. Enhanced healing of rat calvarial defects with MSCs loaded on BMP-2 releasing chitosan/ alginate/hydroxyapatite scaffolds. PLoS One. 2014;9:e104061. doi:10.1371/journal.pone.0104061

18. Zhang J, Wang C, Wang J, Qu Y, Liu G. In vivo drug release and antibacterial properties of vancomycin loaded hydroxyapatite/chitosan composite. Drug Deliv. 2012;19:264-269. doi:10.3109/1071754 4.2012.704093

19. Venkatesan P, Puvvada N, Dash R, et al. The potential of celecoxib-loaded hydroxyapatite-chitosan nanocomposite for the treatment of colon cancer. Biomaterials. 2011;32:3794-3806. doi:10.1016/j.biomaterials.2011.01. 027

20. Fan J, Bi L, Wu T, et al. A combined chitosan/nano-size hydroxyapatite system for the controlled release of icariin. J Mater Sci Mater Med. 2012;23:399-407. doi:10.1007/s10856-011-4491-4

21. Thein-Han WW, Misra RDK. Biomimetic chitosan-nanohydroxyapatite composite scaffolds for bone tissue engineering. Acta Biomater. 2009;5(4):1182-1197. doi:10.1016/j.actbio.2008.11.025

22. Lan LS, Polak SJ, Poellmann MJ, et al. The effect of BMP-2 on microand macroscale osteointegration of biphasic calcium phosphate scaffolds with multiscale porosity. Acta Biomater. 2010;6:3283-3291. doi:10.1016/j.actbio.2010.02.026 
23. Keeney M, van den Beucken JJ, van der Kraan PM, Jansen JA, Pandit A. The ability of a collagen/calcium phosphate scaffold to act as its own vector for gene delivery and to promote bone formation via transfection with VEGF (165). Biomaterials. 2010;31:2893-2902. doi:10.1016/j.biomaterials.2009.12.041

24. Fan J, Bi L, Jin D, et al. Microsurgical techniques used to construct the vascularized and neurotized tissue engineered bone. Biomed Res Int. 2014;2014:281872. doi:10.1155/2014/281872

25. Fan JJ, Mu TW, Qin JJ, Bi L, Pei G-X. Different effects of implanting sensory nerve or blood vessel on the vascularization, neurotization, and osteogenesis of tissue-engineered bone in vivo. Biomed Res Int. 2014;2014:412570. doi:10.1155/2014/412570

26. Engler AJ, Sen S, Sweeney HL, Discher DE. Matrix elasticity directs stem cell lineage specification. Cell. 2006;126:677-689. doi:10.1016/ j.cell.2006.06.044
27. Li J, Wang Q, Zhi W, et al. Immobilization of salvianolic acid Bloaded chitosan microspheres distributed three-dimensionally and homogeneously on the porous surface of hydroxyapatite scaffolds. Biomed Mater. 2016;11(5):055014. doi:10.1088/1748-6041/11/5/055 014

28. Lin S, Cui L, Chen G, et al. PLGA/ $\beta$-TCP composite scaffold incorporating salvianolic acid B promotes bone fusion by angiogenesis and osteogenesis in a rat spinal fusion model. Biomaterials. 2019;196:109-121.

29. Oryan A, Bigham-Sadegh A, Abbasi-Teshnizi F. Effects of osteogenic medium on healing of the experimental critical bone defect in a rabbit model. Bone. 2014;63:53-60. doi:10.1016/j.bone.2014.02.010

\section{Publish your work in this journal}

The International Journal of Nanomedicine is an international, peerreviewed journal focusing on the application of nanotechnology in diagnostics, therapeutics, and drug delivery systems throughout the biomedical field. This journal is indexed on PubMed Central, MedLine, CAS, SciSearch ${ }^{\mathbb{R}}$, Current Contents ${ }^{\mathbb{R}} /$ Clinical Medicine, $^{-}$
Journal Citation Reports/Science Edition, EMBase, Scopus and the Elsevier Bibliographic databases. The manuscript management system is completely online and includes a very quick and fair peer-review system, which is all easy to use. Visit http://www.dovepress.com/ testimonials.php to read real quotes from published authors. 